\title{
Conceptos básicos e importancia de los biocomparables en enfermedades automediadas
}

\section{Basic concepts and importance of biocomparables in self-mediated diseases}

\author{
Rebeca Pérez-Cabeza de Vaca ${ }^{1 *}$, Juan A. Suárez-Cuenca², Tomás Cortés-Espinosa ${ }^{3}$, Josué Mora-Pérez ${ }^{4}$, \\ José L. Zaldívar-Fujigaki ${ }^{5}$ y Carlos Guerrero-Hernández ${ }^{6}$
}

${ }^{1}$ División de Investigación Biomédica, Centro Médico Nacional 20 de Noviembre, Instituto de Seguridad y Servicios Sociales de los Trabajadores del Estado (ISSSTE), Ciudad de México, México; '2Laboratorio de Metabolismo Experimental e Investigación Clínica, Centro Médico Nacional 20 de Noviembre, Instituto de Seguridad y Servicios Sociales de los Trabajadores del Estado (ISSSTE), Ciudad de México, México; ${ }^{3}$ Servicio de Gastroenterología, Centro de Enfermedad Inflamatoria Intestinal, Centro Médico Nacional 20 de Noviembre, Instituto de Seguridad y Servicios Sociales de los Trabajadores del Estado (ISSSTE), Ciudad de México, México; ${ }^{4}$ Departamento de Investigación Clínica, Centro Médico ASSET, Ciudad de México, México; ${ }^{5}$ Departamento de Dermatología, Centro Médico Nacional 20 de Noviembre, Instituto de Seguridad y Servicios Sociales de los Trabajadores del Estado (ISSSTE), Ciudad de México, México; ${ }^{6}$ Stowers Institute for Medical Research, Kansas City, Missouri, USA

\section{Resumen}

Se ha denominado medicamentos biotecnológicos a aquellos que contienen proteínas obtenidas a partir de un sistema biológico, cómo células vivas en cultivo; se distinguen de los medicamentos tradicionales que son producidos mediante síntesis química, conocidos como farmacoquímicos. Recientemente se han introducido sustitutos de estos medicamentos biotecnológicos innovadores (también denominados biofarmacéuticos) por el vencimiento de sus registros de patentes, que comúnmente se conocen como biocomparables. Debido a su complejidad molecular intrínseca de producción en sistemas vivos abiertos, estas copias son similares, pero no idénticas, al medicamento de referencia, y por ello se denominan medicamentos biocomparables. A partir de 2009, los Estados Unidos de América y la Unión Europea estipulan las guías que regulan rigurosamente el uso y la comercialización de estas moléculas. En México, en 2014 se estableció la NOM-257-SSA1-2014, en materia de medicamentos biotecnológicos. Sin duda, la inserción de los medicamentos biocomparables en el mercado tiene como intención favorecer el acceso a ellos de sectores más amplios de población; sin embargo, deben anteponerse rigores que garanticen la máxima eficacia terapéutica y la óptima seguridad de los pacientes, incluyendo así pruebas de bioequivalencia y farmacovigilancia estrecha. Es definitivo que el aumento de los medicamentos biotecnológicos en el mercado puede tener un alto impacto en la prevención, el diagnóstico, el control y el tratamiento de enfermedades, por lo que los medicamentos biocomparables representan una opción segura de acceso a tratamientos innovadores para los pacientes con enfermedades crónicas no transmisibles, como las autoinmunes, y ofrecen una potencial optimización de los recursos del sector salud en México y en todo el mundo.

Palabras clave: Biocomparables. Medicamentos biotecnológicos. Medicamento biocomparable. Anticuerpos. Autoinmune.

Correspondencia:

${ }^{*}$ Rebeca Pérez-Cabeza de Vaca

E-mail: esderebk@gmail.com

2696-6867 / @ 2021 Permanyer. Éste es un artículo open access bajo la licencia CC BY-NC-ND (http://creativecommons.org/licenses/by-nc-nd/4.0/).

Fecha de recepción: 02-07-2021

Fecha de aceptación: 03-11-2021

DOI: 10.24875/IMIDS.M21000013
Disponible en internet: 22-02-2022

Rev Mex Enferm Inflam Inmunomed. 2021;95(4):91-97

www.IMIDsMexico.com 


\section{Abstract}

Biotechnological medicines have been called as those containing proteins obtained from a biological system, as living cells in culture; they are distinguished from traditional medicines that are produced through a chemical synthesis, known as pharmacochemicals. Recently, substitutes have been introduced to these innovative biotechnological medicines (also called biopharmaceuticals) due to the expiration of their patent registrations, which have commonly been called biocomparable. Due to their intrinsic molecular complexity of production in open living systems, these copies are similar but not identical to the reference medicinal product and are therefore called biocomparable medicinal products. As of 2009, the United States and the European Union have established guidelines that strictly regulate the use and commercialization of these molecules. In Mexico, it is at 2014 that NOM-257-SSA1-2014 is established, in the field of biotechnological medicines. Undoubtedly, the insertion of biosimilar medicines in the market is intended to promote the access of wider sectors of the population to these medicines; however, rigors should be put in place to ensure maximum therapeutic efficacy and optimal patient safety, including bioequivalence and close pharmacovigilance testing. It is definitive that the increase of biotechnological medicines in the market can have a high impact on the prevention, diagnosis, control and treatment of diseases, so biocomparable medicines represent a safe option of access to innovative treatments for patients of chronic- non-communicable diseases such as autoimmune and offer a potential optimization of the resources of the health sector in Mexico and worldwide

Keywords: Biopharmaceuticals. Biotechnological medicines. Biocomparable drugs. Antibodies. Autoimmune.

\section{Introducción}

Se ha denominado medicamentos biotecnológicos a aquellos que contienen proteínas obtenidas a partir de un sistema biológico, como lo son las células vivas en cultivo; se distinguen de los medicamentos tradicionales, que son producidos mediante síntesis química, conocidos como farmacoquímicos. El primer medicamento biotecnológico que se desarrolló en el mundo fue la insulina. Actualmente, el $35 \%$ de las sustancias innovadoras en México están relacionadas con moléculas biocomparables, lo que representa importantes ahorros para la economía de las familias y el sector público'.

\section{Definiciones}

Derivado de un rápido crecimiento en la síntesis, la distribución y el uso terapéutico de moléculas biocomparables, junto con la necesidad de una legislación y una definición puntual del concepto de biotecnológico acorde con la regulación internacional, en México se estipularon las siguientes definiciones por parte de la Comisión Federal para la Protección de Riesgos Sanitarios (COFEPRIS), al mismo tiempo que la Ley General de Salud Mexicana se modificaba para contemplar el registro de estos medicamentos para su utilización en nuestro país:

- Medicamento biotecnológico: sustancia producida por medio de biotecnología molecular, con efectos ya sea para la prevención, el tratamiento o la rehabilitación, y presentada en forma farmacéutica. Se identifica como tal dadas su actividad farmacológica y sus propiedades físicas, químicas y biológicas².

- Medicamento biotecnológico innovador: aquel desarrollado originalmente hacia un blanco molecular que ha demostrado eficacia y seguridad para su uso clínico.

- Medicamento biotecnológico biocomparable: medicamento biotecnológico no innovador que demuestre ser biocomparable en términos de seguridad, calidad y eficacia al medicamento biotecnológico de referencia, este nombre es solo utilizado en México, en el resto del mundo son conocidos como medicamentos biocomparables.

- Medicamento biotecnológico de referencia: medicamento biotecnológico innovador que sea reconocido como tal por la Secretaría de Salud y que se utiliza como referencia para el registro de medicamentos biotecnológicos.

A diferencia de los medicamentos convencionales, también llamados farmoquímicos o moléculas pequeñas, los biotecnológicos son moléculas complejas; tanto es así que no existe la posibilidad de equivalencia absoluta entre los originales y sus medicamentos biocomparables. Es relevante establecer las diferencias entre ellos, que se resumen en la tabla 1, así como las principales propiedades que diferencian a los farmacoquímicos de los biocomparables. 
Tabla 1. Comparación entre farmoquímicos y medicamentos biocomparables. Se deben identificar las principales diferencias entre farmoquímicos y biocomparables, ya que químicamente las propiedades les confieren indicaciones y seguimiento terapéutico particular ${ }^{10}$

\begin{tabular}{|l|l|}
\hline Farmoquímicos & Biocomparables \\
\hline Producidos por síntesis química & Producidos en un sistema biológico, células vivas en cultivo \\
\hline Fórmulas de bajo peso molecular & Moléculas de alto peso molecular \\
\hline Estructura bien definida & Estructuras complejas, con heterogeneidad \\
\hline Síntesis dependiente del proceso de elaboración & Dependientes del proceso de síntesis \\
\hline En su gran mayoría estables & $\begin{array}{l}\text { Inestables, más sensibles a condiciones externas, como cambios } \\
\text { de temperatura }\end{array}$ \\
\hline $\begin{array}{l}\text { Caracterizadas completamente desde el punto de vista químico } \\
\text { y atómico }\end{array}$ & $\begin{array}{l}\text { Debido a su heterogeneidad, es complicado dar una precisa } \\
\text { caracterización atómica y estructural }\end{array}$ \\
\hline La gran mayoría no tienen un efecto inmunogénico & Poseen propiedades que generan respuestas inmunogénicas
\end{tabular}

\section{Desarrollo histórico de los medicamentos biocomparables}

La European Medicines Agency (EMA) y la Food and Drug Administration (FDA) han marcado la pauta en el tema a lo largo de la historia, y en México, la COFEPRIS ha iniciado la regulación necesaria para una buena práctica clínica, desde el año 2009.

La EMA publicó los primeros borradores para indicar las "guías" para el manejo de productos medicinales biológicos similares, considerados el primer marco regulatorio para la síntesis, el manejo y la comercialización de biocomparables autorizados, estableciendo con ello el punto de referencia con sus estrictas directrices para este nuevo ámbito biofarmacéutico. En primer lugar, Europa aprueba el primer biocomparable, la somatropina (Omnitrope $\left.{ }^{\circledR}\right)$, para la venta al público, seguida de Australia, que adopta las directrices de la EMA sobre biocomparables de la Unión Europea y realiza posteriormente modificaciones en el año 2013. La promulgada Ley de Protección al Paciente y Cuidado de Salud Asequible (también conocida como Obamacare) se da a la par en los Estados Unidos de América con la intención de iniciar la aprobación por parte de la FDA de biocomparables e intercambiables, y de ahí la Unión Europea publica la primera revisión de sus directrices de 2005. La EMA abre el camino publicando el primer documento regulatorio, que describía las diferencias en las materias primas y la fabricación de productos de referencia y de biocomparables.

Con base en el Primer Proyecto de Ley de Competencia e Innovación de Precios de Productos Biológicos de 2007 (Biologics Price Competition and
Innovation Act), aprobado por el Congreso de los Estados Unidos de América y promulgada al año siguiente como parte de Obamacare ${ }^{3}$, la Organización Mundial de la Salud publica una guía para los estándares de medicamentos biocomparables, que serán reconocidos en todo el mundo ${ }^{4}$. De ahí, países como Canadá aprueban su primer biocomparable, la somatropina (Omnitrope ${ }^{\circledR}$, para la venta, y Health Canada finaliza sus propias pautas regulatorias al año consecutivo. En la región de Asia, Japón publica sus directrices reguladoras de biocomparables, basadas en las directrices de la Unión Europea, y en marzo de 2009 se aprueba el uso su primer biocomparable, la somatropina $^{5}$. Unos años más tarde, la FDA publica un borrador de documento de orientación sobre biocomparables, estableciendo los requisitos para demostrar la comparabilidad de los biocomparables con sus productos de referencia, esto debido a que las patentes de los productos originales estaban por vencer y comenzaría una inserción de los productos biocomparables. Es en el año 2014 cuando en los Estados Unidos de América se aprueba el primer biocomparable, el filgrastim (Zarxio $\left.{ }^{\circledR}\right)$. De manera más reciente, en 2015, la EMA publicó su guía revisada sobre biocomparables ${ }^{6,7}$.

\section{Marco regulatorio de los medicamentos biocomparables en México}

Los medicamentos biotecnológicos son moléculas complejas cuyo proceso de fabricación depende de un sistema biológico, abierto, que no permite controlar todas las variables y que de alguna manera 
impide replicar con gran exactitud la sustancia original, por lo que no existe una equivalencia absoluta entre el fármaco original (innovador) y el biocomparable.

En el caso particular de México, con el vencimiento de las patentes de medicamentos biotecnológicos se ha iniciado el desarrollo de sustancias estructuralmente similares y con las cualidades terapéuticas de las moléculas originales. En México, estas sustancias reciben el nombre de medicamentos biocomparables, lo cual potencialmente representa un mayor acceso para los usuarios en función de su coste, considerando siempre la inmunogenicidad y los efectos adversos asociados a este tipo de medicamentos. Recordemos que los biocomparables imitan la acción terapéutica de los medicamentos biotecnológicos, y se producen después de que las patentes de estos últimos vencen. Para que un medicamento candidato a biocomparable pueda considerarse como tal, debe cumplir con una serie de criterios establecidos en la Norma Oficial Mexicana (NOM) 177; hoy en día, solo existen 13 medicamentos biocomparables registrados ante la COFEPRIS hasta el año $2019^{8}$.

En México, el titular de la COFEPRIS ha señalado que los medicamentos biocomparables representan una opción para el Sistema Nacional de Salud, ya que generan ahorros en la atención de pacientes con enfermedades no transmisibles, como la diabetes mellitus, la obesidad y algunos padecimientos cardiovasculares que han crecido en años recientes. El Senado de la República ha mencionado que este tipo de fármacos son la medicina del futuro, e históricamente la NOM 257-SSA1-2014 ha permitido garantizar la calidad en 59 productos biotecnológicos autorizados y sumar 28 nuevos productos con el más estricto estándar de calidad.

Dentro del marco regulatorio para los biocomparables en México se han establecido diferentes leyes y reglamentos conforme al siguiente desarrollo cronológico:

- En 11 de junio de 2009, por Decreto se realiza una Adición a la Ley General de Salud, artículo 222 Bis, bajo el siguiente concepto: «Para efectos de esta Ley, se considera medicamento biotecnológico toda sustancia que haya sido producida por biotecnología molecular, que tenga efecto terapéutico, preventivo o rehabilitatorio, que se presente en forma farmacéutica, que se identifique como tal por su actividad farmacológica y propiedades físicas, químicas y biológicas. Los medicamentos biotecnológicos innovadores podrán ser referencia para los medicamentos biotecnológicos no innovadores, a los cuales se les denominará biocomparables. La forma de identificación de estos productos será determinada en las disposiciones reglamentarias para garantizar a los médicos y a los pacientes que no se realizará el intercambio entre biotecnológicos innovadores, o entre biotecnológicos innovadores y no innovadores hasta en tanto el médico lo prescriba.»

- En 2011 se reforman e incluyen los conceptos del Reglamento de Insumos para la Salud, artículos 87 y 167.

- Posteriormente se reforma la Ley General de Salud (marco general para medicamentos biotecnológicos y biocomparables, 19 de octubre de 2011).

- Se publica la Norma de Emergencia NOM-EM-001-SSA1-2012.

- Se emite el Reglamento de moléculas nuevas, en febrero de 2012.

- Se regula la evaluación clínica de estos medicamentos con la NOM-177-SSA1-2013, considerando los requisitos a que deben sujetarse los terceros autorizados que realicen pruebas de intercambiabilidad y estudios de medicamentos biotecnológicos.

- Se consolida la regulación a través de la NOM-257-SSA1-2014, en materia de medicamentos biotecnológicos ${ }^{9}$.

\section{Terapéutica de los medicamentos biocomparables}

Mediante el desarrollo de la ingeniería genética que permitió la producción de proteínas recombinantes en cantidades suficientes para utilizarlas terapéuticamente, a partir de los años 1970 se inició una transformación en la era de la farmacología. Estos procesos incorporan fragmentos de ADN recombinante, el cual codifica la producción de proteínas específicas dentro de líneas celulares artificiales que han permitido la producción de biofármacos con fines terapéuticos; por ejemplo, los anticuerpos monoclonales ${ }^{10}$.

Hoy en día, los nuevos medicamentos van más allá de las proteínas recombinantes, ya que actualmente contamos con vacunas de ARN mensajero y en el mercado existen moléculas que interfieren en vías de señalización celular a diversos niveles químicos, de origen proteico o de ácidos nucleicos, como pudieran ser los micro-RNA, y también se destaca la tendencia a emplear medicamentos que tengan la capacidad de regular epigenéticamente (grado de regulación superior a los genes, mediante mecanismos de remodelación de cromatina y modificaciones químicas sobre las hebras 
de ADN) la expresión de genes y, por consecuencia, de la síntesis de proteínas específicas, siendo cada día más amplias la variedad y la gama de posibilidades terapéuticas, y con ello un mayor impacto en cuanto a beneficio del tratamiento de enfermedades complejas, entre ellas las autoinmunes ${ }^{11,12}$.

El surgimiento de toda la regulación y la comercialización de medicamentos biotecnológicos ha beneficiado a millones de pacientes en todo el mundo para el tratamiento de algunas enfermedades, como la enfermedad inflamatoria intestinal (EII), algunos tipos de cáncer, enfermedades autoinmunes, deficiencias genéticas y metabólicas del crecimiento, problemas de reproducción, diabetes dependiente de insulina y diversas enfermedades con un componente de inflamación crónica. Con el paso de los años, los medicamentos biotecnológicos han demostrado su eficacia y seguridad en la Ell en numerosos estudios clínicos. En 1998 se aprobó la primera terapia biotecnológica para el tratamiento de la enfermedad de Crohn y en 2006 para la colitis ulcerosa crónica idiopática. El infliximab (Remicade ${ }^{\circledR}$ ) fue el primer anticuerpo monoclonal de tipo quimérico aprobado para estas indicaciones; después lo fue el anticuerpo monoclonal humano adalimumab (Humira $\left.{ }^{\circledR}\right)$, seguido por el fragmento del anticuerpo monoclonal humanizado certolizumab pegilado $\left(\right.$ Cimzia $\left.{ }^{\circledR}\right)$ y, finalmente, el anticuerpo monoclonal humano golimumab (Simponi ${ }^{\circledR}$ ) para la colitis ulcerosa crónica idiopática. Todos ellos inhiben el factor de necrosis tumoral alfa y han demostrado tener un perfil de eficacia y seguridad aceptable en estudios clínicos especíicos en la Ell10.

Tal como se ha señalado, tras el vencimiento de las patentes de los medicamentos innovadores han surgido alternativas conocidas como medicamentos biocomparables, definidos como los medicamentos no innovadores que han demostrado calidad, eficacia y seguridad comparables a las del medicamento innovador. Los biocomparables pretenden ser estructuralmente idénticos a los medicamentos de referencia, pero ofreciendo un mejor costo-beneficio para el paciente y la institución de salud.

Es relevante destacar que el término «biocomparable» es sinónimo de «biosimilar», y que a diferencia de los medicamentos genéricos, las proteínas sintentizadas en estos sistemas de células vivas en cultivo, como los anticuerpos monoclonales, son moléculas estructuralmente más complejas y de mayor peso molecular, por lo que no es posible alcanzar la "equivalencia absoluta» entre el medicamento innovador y el biocomparable. De ello se desprende la regulación de la NOM-177-SSA1-2013 y que los estudios de biocomparabilidad sean necesarios para demostrar la eficacia y la seguridad en este tipo de medicamentos y su uso en población abierta.

En el caso de los biocomparables, fue a partir de 2004 que el interferón alfa, la insulina recombinante y la hormona del crecimiento perdieron su patente en todo el mundo, por lo que se hizo necesaria la regulación de todos los biotecnológicos emergentes. Las agencias regulatorias, como la EMA y la FDA, y la Organización Mundial de la Salud, cuentan con guías para regular estos medicamentos esencialmente en su caracterización fisicoquímica, eficacia clínica, seguridad e inmunogenicidad, así como su farmacovigilancia.

En relación con el uso clínico, no es posible considerar a los medicamentos biocomparables como intercambiables, ya que, como hemos expuesto, se trata de combinaciones moleculares heterogéneas que requieren el sustento de las pruebas de intercambiabilidad suficientes para su prescripción. Al menos, en la Unión Europea no se consideran intercambiables directos y su acción clínica no se puede extrapolar de aquella producida por los medicamentos innovadores ${ }^{13}$.

En la Unión Europea, de 2006 a 2010 se registraron y aprobaron 14 medicamentos biocomparables ${ }^{14}$. En México se han reconocido oficialmente 13 medicamentos biocomparables y la COFEPRIS, en su última actualización de medicamentos biocomparables, publicó una lista de autorizaciones para uso en seres humanos ${ }^{8}$, las cuales se resumen en la tabla 2.

A la fecha, en nuestro país existen 72 medicamentos biotecnológicos que pueden comercializarse debido a que cuentan con el registro sanitario ante la autoridad local en el rubro: la COFEPRIS. Muchos cuentan con altos registros de eficacia en el tratamiento de enfermedades, y con ello un concomitante aumento en el número de medicamentos biotecnológicos que obtienen el registro sanitario, generando así terapias más eficaces para más enfermedades cada vez; sin embargo, aún existe un obstáculo importante para que el total de la poblacional pueda tener acceso a ellos,y este sin duda es su costo ${ }^{15}$.

Se estima que, para el año 2030, alrededor del 70\% de la población mexicana padecerá diabetes, y el aumento de adultos mayores se extrapola a 15 millones (lo cual se traducirá en un enorme gasto público en el sector salud). La COFEPRIS ha dedicado grandes esfuerzos a impulsar la producción de medicamentos biocomparables que buscan resarcir y compensar este potencial escenario sanitario, generar una 
Rev Mex Enferm Inflam Inmunomed. 2021;95(4)

Tabla 2. Listado de medicamentos biocomparables autorizados por COFEPRIS en México durante el año 2019

\begin{tabular}{|l|l|l|}
\hline Denominación genérica & Laboratorio de producción & Indicación terapeútica \\
\hline Filgastrim & SANDOZ S.A. de C.V. & Neutropenia \\
\hline Filgastrim & PROBIOMED S.A. de C.V. & Neutropenia \\
\hline Eritropoyetina alfa & PROBIOMED S.A. de C.V. & Antianémico \\
\hline Folitropina alfa & Laboratorio Corne S.A. de C.V. & \\
\hline Infliximab & Celtrion Inc y Pfizer & Artritis, Ell \\
\hline Interferón alfa & PROBIOMED S.A. de C.V. & Antiviral \\
\hline Interferón beta & PROBIOMED S.A. de C.V. & Esclerosis múltiple \\
\hline Interferón beta & Laboratorio PISA S.A. de C.V. & Esclerosis múltiple \\
\hline Insulina glargina & Laboratorio PISA S.A. de C.V. & Diabetes Mellitus \\
\hline Insulina glargina & Eli Lily y Compañ́a de México S.A. de C.V. & Diabetes Mellitus \\
\hline Insulina glargina & Wockhandt Limited & Diabetes Mellitus \\
\hline Rituximab & Laboratorio PISA S.A. de C.V. & Linfoma No Hodgking \\
\hline Somatropina & SANDOZ S.A. de C.V. & Alteraciones del crecimiento \\
\hline Trastuzumab & Pfizer & Cáncer de mama y estómago \\
\hline
\end{tabular}

competencia en el mercado y abatir los precios de las medicinas producidas mediante biotecnología. Finalmente, pero no menos transcendental, es sin duda la tarea de los fabricantes de los medicamentos biotecnológicos y biocomparables en el ejercicio de la farmacovigilancia a largo plazo. Los eventos secundarios deben reportarse al portal de COFEPRIS (www.cofepris.gob.mx), así como recomendar el seguimiento estricto de los médicos que los prescriben a los pacientes, esencialmente por los grados de inmunogenicidad y algunos de los efectos secundarios asociados este tipo de terapéutica, siendo una responsabilidad por parte de todos los profesionales de la salud involucrados con su uso.

\section{Financiamiento}

La presente investigación no ha recibido ninguna beca específica de agencias de los sectores público, comercial o sin ánimo de lucro.

\section{Conflicto de intereses}

Los autores declaran no tener conflictos de intereses.

\section{Responsabilidades éticas}

Protección de personas y animales. Los autores declaran que para esta investigación no se han realizado experimentos en seres humanos ni en animales.

Confidencialidad de los datos. Los autores declaran que en este artículo no aparecen datos de pacientes.

Derecho a la privacidad y consentimiento informado. Los autores declaran que en este artículo no aparecen datos de pacientes.

\section{Referencias}

1. Comisión Federal para la Protección contra Riesgos Sanitarios. Biocomparables, opción para reducir costos en el Sistema Nacional de Salud. Citado: 20/10/2021. Disponible en: https://www.gob.mx/cofepris/articulos/ biocomparables-opcion-para-reducir-costos-en-el-sistema-nacional-de-salud-136953?idiom=es

2. Reglamento de insumos para la salud, Ley General de Salud. 2009. Citado: 26/10/2021. Disponible en: http://dof.gob.mx/nota_detalle.php?codigo $=4864792 \&$ fecha $=04 / 02 / 1998$

3. Biologics Price Competition and Innovation Act of 2007 (2007 S. 1695). 2007. Citado: 20/10/2021. Disponible en: https://www.govtrack.us/congress/bills/110/s1695

4. WHO guidelines on biosimilars: case studies and discussion highlights Reports. Home GaBI online Generics and Biosimilars Initiative. Gabionlinenet. 2012. Citado: 20/10/2021. Disponible en: http://www.gabionline. net/Reports/WHOguidelinesonbiosimilarscasestudiesand- discussionhighlights

5. Sandoz receives approval for first ever Japanese biosimilar. 2009. Citado: 20/10/2021. Disponible en: http://www.sandoz.com/media center/ press_releases_news/global_news/sandoz_receives_approval_for_ first- ever_japanese_biosimilar.shtml 


\section{R. Pérez-Cabeza de Vaca et al.: Conceptos básicos e importancia de los biocomparables}

6. European Medicines Agency. Citado: 25/10/2021. Disponible en: http:// www.biosimilarnews.com/emapublishedtherevisedbiosimilars guideline

7. Biosimilar regulations/guidelines in the EU and USA Citado: $31 / 10 / 2021$ Disponible en: https://www.researchgate.net/publication/303690283/figure/fig1/AS:368151851225090@1464785649481/Biosimilar-regulations-guidelines-in-the-EU-and-USA-Boxed-items-represent-those-timeline.png

8. Secretaría de Salud/COFEPRIS. Listado de medicamentos biotecnológicos biocomparables. Citado: 31/10/2021. Disponible en: https:/ www.gob.mx/cms/uploads/attachment/file/468862/Listado_de_Medicamentos_Biotecnol_gicos_Biocomparables__Versi_n_6_11-06-2019.pdf

9. Norma Oficial Mexicana NOM-257-SSA1-2014, En materia de medicamentos biotecnológicos. Citado: 1/11/2021. Disponible en: http://dof.gob. $\mathrm{mx} /$ nota_detalle.php?codigo $=5375517 \&$ fecha $=11 / 12 / 2014$
10. Mayoral-Zavala A, Esquivel-Aguilar A, del Real-Calzada CM, Gutiérrez-Grobe Y, Ramos-García J, Rocha-Ramírez JL, et al. Actualización sobre los medicamentos biocomparables en la enfermedad inflamatoria intestinal: posición y recomendación en México. Rev Gastroenterol Mex. 2018;83:414-23.

11. Bonini S, Bonini M. Biosimilars and drug development in allergic and immunologic diseases. J Allergy Clin Immunol. 2017;139:1461-4.

12. Declerck PJ. Biologicals and biosimilars: a review of the science and its implications. Generics and Biosimilars Initiative Journal (GaBI Journal). 2012;1:13-6.

13. Dranitsaris G, Amir E, Dorward K. Biomsimilars of biological drugs therapies, regulatory, clinical and commercial considerations. Drugs. 2011;71:1527-36.

14. McCamish M, Woollett G. Worldwide experience with biosimiliar development. MAbs. 2011;3:209-17.

15. López-Silva C. México retoma el liderazgo regulatorio sobre medicamentos biotecnológicos y biocomparables. Gac Med Mex. 2012;148:83-90. 